\title{
APPLICATION OF TWO-POINT PADÉ APPROXIMANTS TO SOME SOLID STATE PROBLEMS
}

PING SHENG

A simple application of the two-point Pade approximants to the problem of polaron [5] is presented in the following. A conduction electron in a polar crystal will polarize and distort the ion lattice in its neighborhood. The lattice polarization, in turn, acts back on the electron and lowers its energy. The system of electron and its accompanying self-consistent polarization field is called a polaron. The Hamiltonian for the polaron is [3]

$$
\begin{aligned}
& H=\sum_{k} \frac{h^{2} k^{2}}{2 m} c_{k}{ }^{+} c_{k}+h \Omega \sum_{q}\left(a_{q}{ }^{+} a_{q}+\frac{1}{2}\right)+H_{1}, \\
& H_{1}=i h \Omega\left(\frac{4 \pi \alpha}{V}\right)^{1 / 2}\left(\frac{h}{2 m \Omega}\right)^{1 / 4} \sum_{k, q} \frac{1}{|q|}\left(a_{q}{ }^{+}-a_{-q}\right) c_{k-q}^{+} c_{k} .
\end{aligned}
$$

Here $\Omega$ is the optical phonon frequency, $h$ is the Planck's constant, $\alpha=e^{2} / 2\left(1 / \epsilon_{\infty}-1 / \epsilon_{0}\right) \quad\left(2 m / \Omega h^{3}\right)^{1 / 2}$ is the dimensionless polaron coupling constant, $e$ is the charge of the electron, $m$ is the conductionbased mass, $\epsilon_{0}$ and $\epsilon_{\infty}$ are the static and optical dielectric constants of the solid respectively, $a_{q}\left(a_{q}{ }^{+}\right)$is an operator which destroys (creates) a longitudinal optical phonon of wavevector $q$, and $c_{\boldsymbol{k}}\left(c_{k}{ }^{+}\right)$is an operator which destroys (creates) an electron of momentum $k$. There are two limits in which an approximate solution of the Schrödinger equation for the Hamiltonian (1) can be obtained: the weak-coupling limit $(\alpha \ll 1)$ and the strong-coupling limit $(\alpha \gg 1)$. The weakcoupling expansions of the ground state energy and effective mass of the polaron are [5] :

$$
E_{0} / h \Omega=-\alpha-0.01592 \alpha^{2}-0.008765 \alpha^{3}+\cdots
$$

and

$$
m / m^{*}=1-\frac{\alpha}{6}+0.02263 \alpha^{2}+\cdots,
$$

where $E_{0}$ is the ground state energy and $\mathrm{M}^{*}$ is the effective mass of the polaron. In the strong coupling limit, we have the following expansions due to [1] and [2] respectively:

Research sponsored by the National Science Foundation, Grant No. GP-16147 A \#1. 


$$
E_{0} \not h \Omega=-0.1088 \alpha^{2}-2.83
$$

and

$$
m^{*} / m=0.02 \alpha^{4}-1.01 \alpha^{2}
$$

In order to obtain the value of $E_{0}$ and $m^{*}$ for intermediate values of $\alpha$, we construct two-point Padé approximants to these weak and strong coupling expansions as follows. Instead of determining all the coefficients $\left(a_{0} \cdots a_{N}, b_{1} \cdots b_{M}\right)$ of a Padé approximant

$$
P([M, N] ; Z)=\frac{a_{0}+a_{1} Z+a_{2} Z^{2}+\cdots+a_{N} Z^{N}}{1+b_{1} Z+\cdots+b_{M} Z^{M}},
$$

from the expansion around $Z=0$, now only half of the equations determining the coefficients will come from the expansion around $Z=0$. The remaining equations are determined by the expansion around $Z=\infty$. The results of such a fit are displayed in [5]. The energy vs. momentum relation for the polaron has also been similarly determined. In order to determine the accuracy of such fits, we construct the two-point Padé approximants of same order to fit Feynman's variational results at the weak and the strong coupling limits. The result indicates that the error for $E_{0}(\alpha)$ is at most 6-7\% and the error for $\operatorname{lnm}^{*}$ is at most $21 \%$.

The method of two-point Padé approximants has also been used to determine the melting density of an electron lattice [4]. Due to the presence of logarithmic terms in the high density expansion of the ground state energy, the two-point Padé approximants are constructed to fit $r_{s}{ }^{4}\left(\partial^{2} E_{0} / \partial r_{s}^{2}\right)$, where $r_{s}=(4 \pi / 3 \cdot \text { density })^{-1 / 3} \cdot(\text { Bohr radius })^{-1}$. When the Padé approximants are twice integrated, the resultant $E_{0}\left(r_{s}\right)$ has a van der Waals loop at $r_{s} \cong 14.3$ provided that the low density expansion of $E_{0}$ has a term of the form $-4 / 35 t r_{s}^{-5 / 2}$, where $-2 \leqq t \leqq 7$ is a constant.

\section{REFERENCES}

1. G. R. Allcock, On the Polaron Rest Energy and Effective Mass, Adv. in Phys. 5 (1956), 412-451.

2. R. P. Feynman, Slow Electrons in a Polar Crystal, Phys. Rev. 97 (1965), 660-665.

3. H. Frölich, Electrons in Lattice Fields, Adv. in Phy. 3 (1954), 325-361.

4. A. Isihara and E. W. Montroll, A Note on the Ground State Energy of an Assembly of Interacting Electrons, Proc. Nat. Acad. Sci. 68 (1971), 3111-3115.

5. P. Sheng and J. D. Dow, Intermediate Coupling Theory: Padé Approximants for Polarons, Phys. Rev. B4 (1971), 1343-1359.

Institute for Advanced Study, Princeton, New Jersey 08540 